\title{
Features of dynamic loading of transport vehicles in intensive operation
}

\author{
Andrey Eliseev ${ }^{1, *}$, Sergey Eliseev ${ }^{1}$, and Alexey Orlenko ${ }^{2}$ \\ ${ }^{1}$ Irkutsk State Transport University, 664074 Irkutsk, Russia \\ ${ }^{2}$ Krasnoyarsk Institute of Railway Transport, branch of the Irkutsk State Transport University, 660028 \\ Krasnoyarsk, Russia
}

\begin{abstract}
The paper proposes a method to construct mathematical models of technical objects exposed to intense vibrational interactions, which is typical for assemblies of transport vehicles. A technique has been developed to construct structural mathematical models in the form of structural diagrams, which are dynamically equivalent to automatic control systems. Analytical tools have been adopted from the theory of automatic control. The paper demonstrates the possibilities of changing dynamic properties of technical objects, design schemes of which are represented by mechanical oscillatory systems. Changes in the dynamic effects arising from the introduction of additional inertial couplings have been estimated. Effects of new dynamic properties have been considered. Analytic relations have been proposed for detailed estimates.
\end{abstract}

\section{Introduction}

Maintaining operational reliability and safety of railway rolling stock is one of the most important and relevant scientific and engineering problems, which have been reflected in studies of Russian scientists [1-3]. The development prospects of transport systems require that a special attention is given to developing a methodological basis for dynamics and operation of vehicles under intense dynamic loading. It is important to assess dynamic states of transport vehicles because this assessment plays an important role in developing principles and specific techniques to be implemented in the systems that ensure the general operational safety of transport vehicles and reliability of their major units and assemblies [4-6].

An increase in speeds and axle loads has a significant effect on the dynamic state of a traction motor. This can be attributed to a change in dynamic stiffness and generation of dynamic bursts produced by a combination of disturbing factors. Some aspects of these issues are considered in [7-10]. The available studies show that motions with increased velocities initiate the development of oscillatory processes with increasing oscillation amplitudes and resulting impact interactions. Passive means of adjusting dynamic states of motor mounting at high speeds of vehicles are not effective enough, especially in situations where natural frequencies and dynamic damping of oscillations are sufficiently close to

\footnotetext{
* Corresponding author: eliseew_aw@irgups.ru
} 
each other. A promising solution in these situations is using motion conversion devices that are capable to widely vary the reduced mass of an object with a controlled dynamic state.

Traction electric motors of electric locomotives are among the technical devices that are subject to a lot of mechanical factors arising from both wheel-rail contacts and interactions with the entire complex mechanical system as a whole, which is determined by structural and engineering characteristics of a locomotive [11-12].

The novelty of this study is in examining scientific foundations underlying mechanisms of dynamic bursts or a certain bunch of oscillatory processes that arise in contacts between the traction motor case and elements connected to the truck frame. The authors put forward the idea of constructing frequency diagrams with families of frequency parameters (partial and natural frequencies, dynamic damping frequencies) constructed according to a parameter to be adjusted. The physical meaning of the burst generation mechanism implies uncontrolled convergence of frequency parameters due to simultaneous action of several external disturbances.

This study focuses on methodological aspects in the development of methods and tools aimed at maintaining the operational safety of locomotive traction motors. The key feature of the approach is that it takes into account possibilities of changing dynamic states of a motor mounting system in transport vehicles by introducing additional couplings. The paper gives a special focus to connectivity between parameters of mounting elements and simultaneous action of several external disturbances so that these aspects are taken into account in the approach.

\section{Problem statement}

Design diagrams representing mechanical oscillatory systems with lumped parameters and several degrees of freedom are used to assess dynamic properties of technical objects, including traction motors. Depending on tasks assigned, traction motors can be represented as mechanical chain systems with two or three degrees of freedom, as well as mechanical oscillatory systems with an object in the form of a solid body on elastic supports, which is in planar motion (Fig. 1). External influences on the traction motor are generated not only as external kinematic disturbances on the side of the bearing surface, but also can be created by irregular electrical loads on the traction motor armature and periodic force disturbances of a different nature [6].

Dynamics of traction motor units can be detailed according to the notions of vibrational effects from the traction motor case on the operation of the commutator-and-brush assembly. The commutator-and-brush assembly is subject to a large number of mechanical factors, among which contacts with surface roughnesses of the armature commutator are of particular importance. At the same time, vibrations from the case of the commutator-andbrush assembly significantly affect the operation of the traction electric motor $[2,11]$.

Figure 1 shows the design diagram of a frame- and axle-mounted traction electric motor. A special feature of the design scheme is the introduction of additional couplings in the mechanical oscillatory system (Fig. 1) in the form of motion conversion devices with reduced masses $L_{1}$ and $L_{2}$. Values of these parameters may change if the following condition is fulfilled:

$$
L_{2}=\alpha L_{1}
$$

where $\alpha$ is the connectivity coefficient for parameters $L_{1}$ and $L_{2}$. It is assumed that $\alpha$ is a positive quantity and can vary in a sufficiently wide range [13]. 


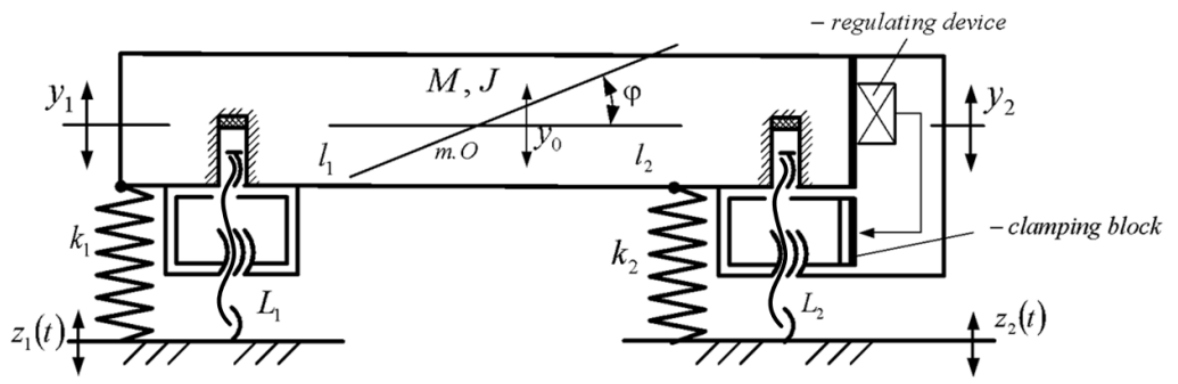

Fig. 1. The design diagram of a frame- and axle-mounted traction electric motor for motion conversion.

The authors are developing an approach within the framework of structural mathematical modeling [14]. The mathematical model of the initial system (Fig. 1) is presented in Figure 2. It is represented as a structural scheme that is dynamically equivalent to the automatic control system.

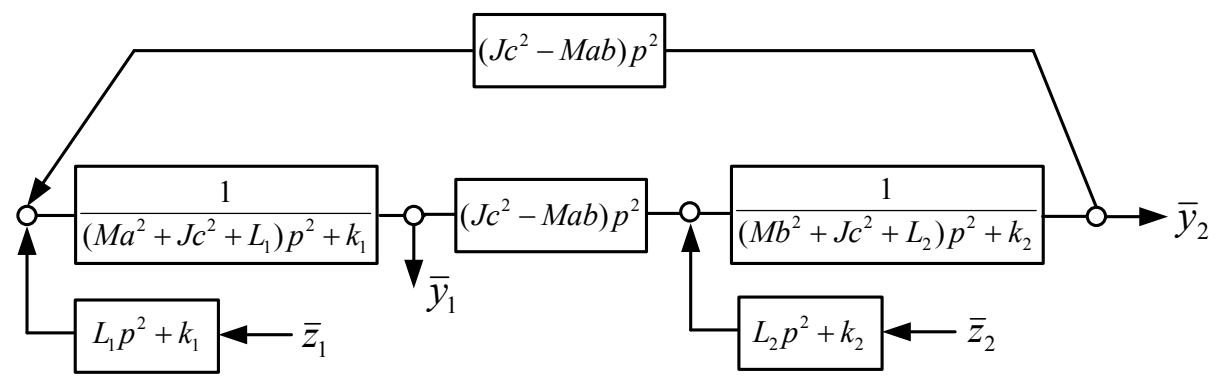

Fig. 2. The structural mathematical model of the initial system taken from Figure 1 with kinematic disturbances.

Figure 2 assumes that $p=j \omega$ as a complex variable; symbol "-_" over the variable means its Laplace transform under zero initial conditions $[15 \div 16]$. The system has two input actions: $\bar{z}_{1}$ and $\bar{z}_{2}$. The dynamic state is estimated with variables $\bar{y}_{1}, \bar{y}_{2} ; M, J$ - weight and moment of inertia of the solid body, respectively; $a, b, c$ - the geometric parameters of the system.

The purpose of the study is to assess the features of a dynamic system, which is simultaneously excited by several disturbances that create some specific dynamic effects.

\section{Mathematical modeling}

Taking into account the simultaneous action of external disturbances, transfer functions of the system can be represented as

$$
\begin{aligned}
& \left.W_{1}(p)\right|_{\bar{z}_{1}=\bar{z}_{2}=\bar{z}}=\frac{\bar{y}_{1}}{\bar{z}}= \\
& =\frac{\left(L_{1} p^{2}+k_{1}\right)\left[\left(M b^{2}+J c^{2}+L_{2}\right) p^{2}+k_{2}\right]+\left(L_{2} p^{2}+k_{2}\right)\left(J c^{2}-M a b\right) p^{2}}{A(p)}
\end{aligned}
$$




$$
\begin{aligned}
& \left.W_{2}(p)\right|_{\bar{z}_{1}=\bar{z}_{2}=\bar{z}}=\frac{\bar{y}_{2}}{\bar{z}}= \\
& =\frac{\left(L_{2} p^{2}+k_{2}\right)\left[\left(M a^{2}+J c^{2}+L_{1}\right) p^{2}+k_{1}\right]+\left(L_{1} p^{2}+k_{1}\right)\left(J c^{2}-M a b\right) p^{2}}{A(p)},
\end{aligned}
$$

where

$$
\begin{aligned}
& A(p)=\left[\left(M a^{2}+J c^{2}+L_{1}\right) p^{2}+k_{1}\right] \cdot\left[\left(M b^{2}+J c^{2}+L_{2}\right) p^{2}+k_{2}\right]- \\
& -\left[\left(J c^{2}-M a b\right) p^{2}\right]^{2}
\end{aligned}
$$

$a, b, c$ are the geometric parameters.

From $(2) \div(4)$ it follows that the system has two frequencies at which resonant phenomena are possible. Frequencies of such processes can be determined from equation (4). In turn, the system for each coordinate $\bar{y}_{1}, \bar{y}_{2}$ may be subject to the dynamic oscillation damping mode. The respective oscillation frequencies can be determined by setting the numerators of the transfer functions (2) and (3) to zero.

Using (1), (2) $\div$ (4) can be transformed as follows

$$
\begin{aligned}
& \left.W_{1}^{\prime}(p)\right|_{L_{2}=\alpha L_{1}}=\frac{\bar{y}_{1}}{\bar{z}}= \\
& =\frac{\left(L_{1} p^{2}+k_{1}\right)\left[\left(M b^{2}+J c^{2}+\alpha L_{1}\right) p^{2}+k_{2}\right]+\left(\alpha L_{1} p^{2}+k_{2}\right)\left(J c^{2}-M a b\right) p^{2}}{A_{1}(p)} \\
& \left.W_{1}^{\prime}(p)\right|_{L_{2}=\alpha L_{1}}=\frac{\bar{y}_{2}}{\bar{z}}= \\
& =\frac{\left(\alpha L_{1} p^{2}+k_{2}\right)\left[\left(M a^{2}+J c^{2}+L_{1}\right) p^{2}+k_{1}\right]+\left(L_{1} p^{2}+k_{1}\right)\left(J c^{2}-M a b\right) p^{2}}{A_{1}(p)}
\end{aligned}
$$

where

$$
\begin{aligned}
& A_{1}(p)=\left[\left(M a^{2}+J c^{2}+L_{1}\right) p^{2}+k_{1}\right] \cdot\left[\left(M b^{2}+J c^{2}+\alpha L_{1}\right) p^{2}+k_{2}\right]- \\
& -\left[\left(J c^{2}-M a b\right) p^{2}\right]^{2}
\end{aligned}
$$

In this case, the partial frequencies of the system are given by expressions

$$
\begin{gathered}
n_{1}^{2}=\frac{k_{1}}{M a^{2}+J c^{2}+L_{1}}, \\
n_{2}^{2}=\frac{k_{2}}{M b^{2}+J c^{2}+\alpha L_{1}},
\end{gathered}
$$

from which it follows that the partial frequency $n_{2}$ depends on the connectivity coefficient $\alpha$. In our case, in contrast to conventional approaches [13, 14], dynamic damping frequencies will no longer coincide with values of partial frequencies, but will be determined from the "zeroing" condition, i.e. setting the numerators of the rational expressions in the corresponding transfer functions to zero. This can be attributed, in particular, to features of solutions to two biquadratic equations. 


$$
\begin{gathered}
p^{4}\left[L_{1}\left(M b^{2}+J c^{2}+\alpha L_{1}\right)+\alpha L_{1}\left(J c^{2}-M a b\right)\right]+p^{2}\left[k_{1}\left(M b^{2}+J c^{2}+\alpha L_{1}\right)+\right. \\
\left.+k_{2}\left(L_{1}+J c^{2}-M a b\right)\right]+k_{1} k_{2}=0 \\
p^{4}\left[\alpha L_{1}\left(M a^{2}+J c^{2}+L_{1}\right)+L_{1}\left(J c^{2}-M a b\right)\right]+p^{2}\left[k_{2}\left(M a^{2}+J c^{2}+L_{1}\right)+\right. \\
\left.+k_{1}\left(\alpha L_{1}+J c^{2}-M a b\right)\right]+k_{1} k_{2}=0 .
\end{gathered}
$$

Thus, the probability of dynamic damping conditions of oscillations will be assessed according to the corresponding roots of equations (10) and (11). In this respect, a graphoanalytical method has some capabilities and could be called a "frequency diagram construction method".

This diagram is constructed as a set of dependency graphs $n_{1}^{2}(\alpha), n_{2}^{2}(\alpha)$, $\omega_{1 \text { dyn }}^{2}(\alpha), \quad \omega_{2 \text { dyn }}^{2}(\alpha), \quad \omega_{1 \mathrm{n} a t}^{2}(\alpha), \quad \omega_{2 \mathrm{n} a t}^{2}(\alpha)$. The dependency graphs $\omega_{1 \text { dyn }}^{2}(\alpha)$ $\omega_{2 \text { dyn }}^{2}(\alpha)$ show relationships between dynamic oscillation damping conditions. Conventional interaction schemes, in which $L_{1}=0, L_{2}=0$ in the coordinate system $y_{1}$ and $y_{2}$, with only a single external disturbance (e.g., $z_{1}(t) \neq 0, z_{2}(t)=0$ ), the dynamic oscillation damping mode can be realized only with respect to the coordinate $\bar{y}_{1}$. In this case, the dynamic damping frequency coincides with the partial frequency $n_{1}$, i.e. $\omega_{1 d y n}^{2}=n_{2}^{2}$. In the case where $z_{1}(t)=0, z_{2}(t) \neq 0$, only one disturbing factor will be involved as well. In this connection, the dynamic damping frequency will also coincide with the corresponding partial frequency, i.e., the condition $\omega_{2 \text { dyn }}^{2}=n_{1}^{2}$ will be satisfied.

With the simultaneous action of two kinematic perturbations, $\bar{z}_{1}=\bar{z}_{2}=\bar{z}$ and $L_{1}=0$, $L_{2}=0$, dynamic damping frequencies can be realized with respect to both coordinates $\bar{y}_{1}$ and $\bar{y}_{2}$, which is defined by expressions:

$$
\begin{aligned}
& \omega_{1 d y n}^{2}=\frac{k_{1} k_{2}}{J c^{2}\left(k_{1}+k_{2}\right)+M b\left(k_{1} b-k_{2} a\right)}, \\
& \omega_{2 d y n}^{2}=\frac{k_{1} k_{2}}{J c^{2}\left(k_{1}+k_{2}\right)+M a\left(k_{2} a-k_{1} b\right)} .
\end{aligned}
$$

Provided that $k_{1} b-k_{2} a=0$, two dynamic damping frequency modes can coincide with each other at a single frequency, which corresponds to certain symmetry relations of system properties (at $a=b$, the condition $k_{1}=k_{2}$ is satisfied; this case needs to be addressed separately).

\section{Estimation of dynamic properties based on the frequency diagram of the system}

The following system parameters are selected to construct a frequency diagram for the model problem. $M=7,000 \mathrm{~kg} ; J=2,000 \mathrm{~kg} . \mathrm{m}^{2} ; a=0.57 ; b=0.43 ; c=0.71 ; k_{1}=1,000$ $\mathrm{kN} / \mathrm{m} ; k_{2}=2,000 \mathrm{kN} / \mathrm{m} ; l_{1}=0.6 \mathrm{~m} ; l_{2}=0.8 \mathrm{~m} ; L_{1}=100 \mathrm{~kg}$.

The dynamic damping frequency at which simultaneous dynamic damping of oscillations will occur is identified by intersection of the dependence graphs $\omega_{1 \text { dyn }}^{2}(\alpha)$ and 
$\omega_{2 d y n}^{2}(\alpha)$ on the frequency diagram (Fig. 3). Two graphs $\omega_{1 d y n}^{2}(\alpha)$ and $\omega_{2 d y n}^{2}(\alpha)$ intersect at point (2), which simultaneously determines the coefficient of connectivity $\alpha$ and a frequency at which simultaneous dynamic damping occurs with respect to coordinates $y_{1}$ and $y_{2}$. Point (1') determines the dynamic damping case only with respect to the coordinate $y_{2}$. In both cases, points (1) and ( $\left.1^{\prime}\right)$ can be projected to the y-axis in parallel to determine the respective dynamic damping frequencies. Moreover, point (2) is an intersection point between the dependency graphs $\omega_{1 \text { dyn }}^{2}(\alpha)$ and $\omega_{2 \text { dyn }}^{2}(\alpha)$, which determines the dynamic damping mode with respect to both coordinates. This occurs at $\alpha=31,205$. The dynamic damping frequency can be found in the same way — by projecting the points to the y-axis. Points (3), (3') and (4) are representative of the effects of a significant change in the dependence graphs $\omega_{1 d y n}^{2}(\alpha)$ and $\omega_{2 d y n}^{2}(\alpha)$, which is associated with the corresponding frequency responses of the system [13].

The frequency diagram (Fig. 3) shows partial, natural and dynamic damping frequencies as a function of $\alpha$ (symbols used on the dependency graphs are explained in Fig. 3). In order to show the system properties, representative points of the graphs are introduced. In this case, points (1), (2), (3), and (4) are considered.

In order to estimate the features of the frequency diagram in the vicinity of points (3) and (3'), point (5) with a value $\alpha=0.93$, marked on the $\mathrm{x}$-axis of the dependence curve $\omega_{1 \mathrm{~d} y n}^{2}(\alpha)$ (Fig. 3), is used. Point (4) corresponds to bends on the dependence curve $\omega_{2 \mathrm{~d} y n}^{2}(\alpha)$. For point (1), which corresponds to $\alpha=2$ (on the $\mathrm{x}$-axis), the frequency diagram reflects specific dynamic properties: both coordinates $\bar{y}_{1}, \bar{y}_{2}$ become equal to zero simultaneously at the same frequency.
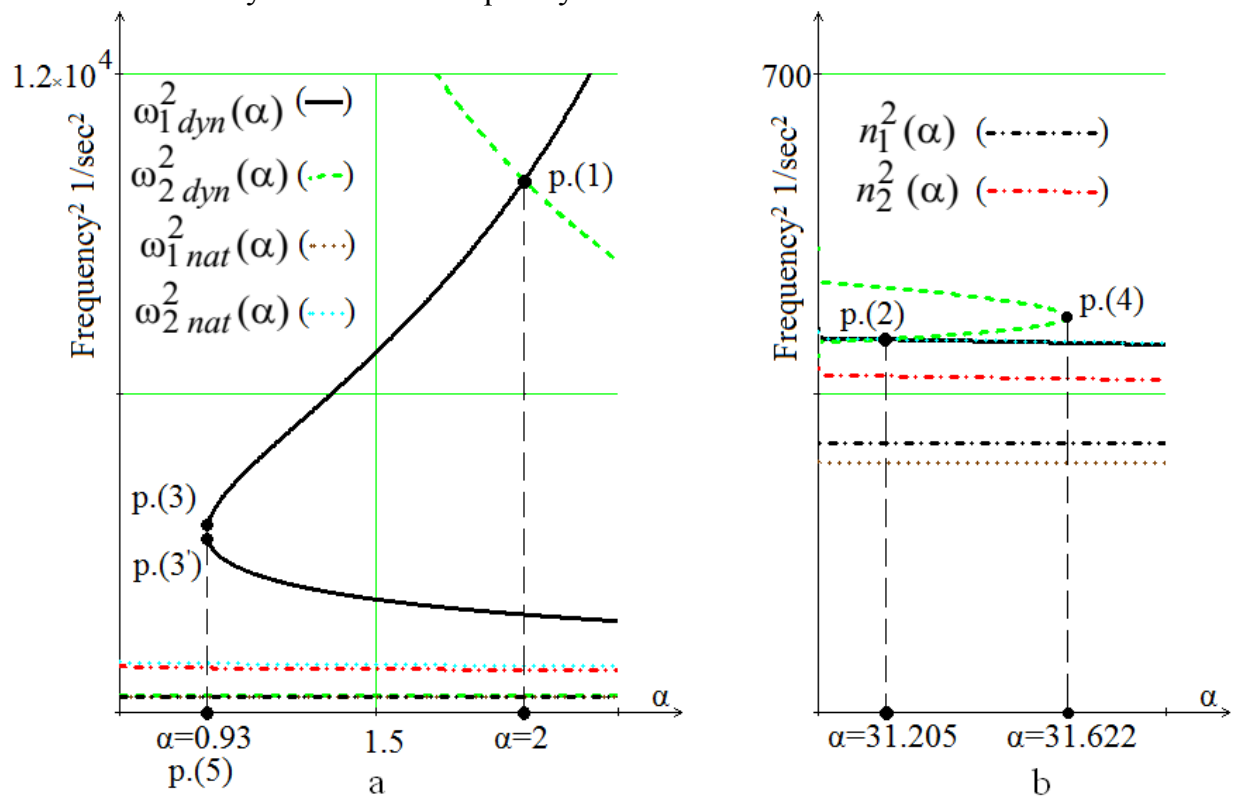

Fig. 3. The frequency diagram for determining dynamic damping modes at $L_{1} \neq 0, L_{2} \neq 0$ and different values of the connectivity coefficient $\alpha$ : a - a range of the y-coordinate $\left(0.1 .2 \times 10^{4}\right)$; $b$ - a range of the $y$-coordinate $(0,700)$. 
Thus, the frequency diagram gives an integral representation of distribution dynamics for characteristic frequencies of the system and the possibilities of realizing certain dynamic effects.

\section{Conclusion}

The authors have developed a method to construct mathematical models aimed at estimating dynamic properties of systems with several degrees of freedom under the combined action of several external in-phase harmonic disturbances. They propose a method for constructing frequency diagrams of mechanical oscillation systems. These methods have been used to study possible dynamic properties of systems under the simultaneous action of two disturbances in dynamic damping modes. Possibilities of generating dynamic damping modes with respect to two coordinates simultaneously, as well as change modes of frequency response with variation of the connectivity coefficient $\alpha$, have been identified. It has been shown that changes in dynamic properties of the system can be obtained by introducing additional links or devices for motion conversion. Physically, the connectivity coefficient determines the ratio between reduced masses of motion conversion devices.

The authors have developed an original method to estimate the potential for generating various instability modes in processes where natural frequencies and dynamic damping of oscillations converge to each other. They have proposed a method based on the construction of frequency diagrams that can be used to find possible frequency zones of unstable operation. It has been shown that dynamic bursts are shown to be predetermined by a combination of disturbing factors. Physically, this phenomenon is equivalent to a change in the reduced dynamic stiffness. Change in the reduced mass, whose value is generated by a special motion conversion mechanism can be controlled, thereby maintaining the level of oscillations of traction motors within a normal range and avoiding collisions of the motor case against stops.

A feasibility study of active mounting systems for traction motors, which are capable to prevent dynamic bursts exceeding the recommended limits in terms of quantity and qualitative parameters, can be proposed as one of further research areas.

\section{References}

1. O.S. Valinsky, Zheleznodorozhnyj transport [Railway transport] 2, 48-51 (2018). (In Russian)

2. A.I. Orlenko, M.N. Petrov, O.A. Teregulov, Kompleksnaja diagnostika tjagovogo jelektrodvigatelja jelektrovoza [Comprehensive diagnostics of locomotive traction electric motor] (OOO Polikom, Krasnoyarsk, 2016). (In Russian)

3. V.V. Kharlamov, P.K. Shkodun, A.V. Dolgova, D.A. Akhunov, Sovershenstvovanie tehnologii diagnostirovanija tehnicheskogo sostojanija kollektorno-shhetochnogo uzla tjagovyh jelektrodvigatelej jelektrovozov [Improvement of diagnistics technologies for operational condition of a collector-and-brush assembly unit of locomotive traction electric motor] (OSTU, Omsk, 2015) (In Russian).

4. A.N. Savoskin, Y.S. Romen, A.A. Akishin, World of Transport and Transportation 13 1 (56), (2015). (In Russian)

5. Y.S. Romen, Zheleznodorozhnyj transport [Railway transport] 11, 62-64 (2017). (In Russian)

6. A.N. Smalev, Candidate of sciences thesis, Omsk, 2011. (In Russian)

7. A. Sladkowski, Rail Transport—Systems Approach (Cham: Springer, 2017). 
8. A. S. Girnik, A. L. Fedyanin, A. V. Kiselev, E, S. Dorokhina, J. Mach. Manuf. Reliab. 46, 240-245 (2017)

9. D. Ya. Antipin, D. A. Bondarenko, O. V. Izmerov, Procedia Engineering 206, 1583-6 (2017).

10. I. Galiev, A. Shiler, A. Kildibekov, I. Kukushkin, Procedia Engineering 100, 1724-31 (2015).

11. S.K. Ismailov, Doctoral thesis, Omsk, 2004. (In Russian)

12. I.I. Galiev, V.A. Nekhaev, V.A. Nikolaev, Metody $i$ sredstva vibrozashhity zheleznodorozhny jekipazhej [Methods and means of vibration protection of railway carriages] (Moscow, 2010). (In Russian)

13. S.V. Eliseev, A.I. Orlenko, V sbornike: Kulaginskie chtenija: tehnika i tehnologii proizvodstvennyh processov [Proceedings of Kulaginskie readings: methods and technologies of production processes], 142-148 (Transbaikal State University Publ., Chita, 2017). (In Russian)

14. S.V. Eliseev, A.I. Artyunin, Prikladnaja teorija kolebanij v zadachah dinamiki linejnyh mehanicheskih sistem [Applied theory of vibrations in problems of linear mechanical systems dynamics] (Nauka Publ., Novosibirsk, 2016). (In Russian)

15. de Silva, Clarence W.,Vibration. Fundamentals and Practice (Boca Raton, London, New York, Washington, D.C.: CRC Press, 2000).

16. I.A. Karnovsky, E. Lebed. Theory of vibration protection. (Switzerland: Springer, 2016). 